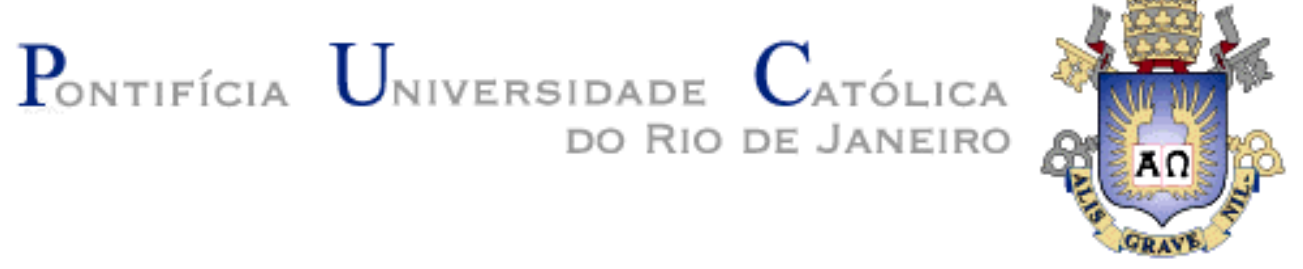

José EURICO de VASCONCELOS FILHO

\title{
Um Modelo de Suporte ao Design Baseado no Rationale: Relacionando Espaço de Problema ao Espaço de Solução no Design
}

\section{TESE De Doutorado}

Tese apresentada ao Programa de Pós-graduação em Informática do Departamento de Informática da PUC-Rio como requisito parcial para a obtenção do título de Doutor em Informática.

Orientadora: prof $^{a}$. Simone Diniz Junqueira Barbosa 


\title{
Pontifícia U Uiversidade Católlica $_{\text {a }}$

\section{José Eurico de Vasconcelos Filho}

\section{Um Modelo de Suporte aO Design Baseado no Rationale:}

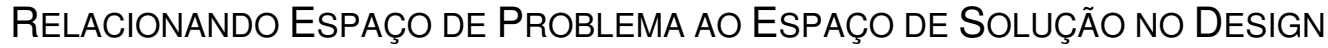

\begin{abstract}
Tese apresentada como requisito parcial para a obtenção do grau de Doutor pelo Programa de Pósgraduação em Informática do Departamento de Informática do Centro Técnico e Científico da PUCRio. Aprovada pela Comissão Examinadora abaixo assinada.
\end{abstract}

Prof ${ }^{a}$. Simone Diniz Junqueira Barbosa

Orientadora

Departamento de Informática - PUC-Rio

Prof. João José Vasco Peixoto Furtado

Coorientador Centro de Ciências Tecnológicas - UNIFOR

Prof $^{\mathrm{a}}$. Clarisse Siekenius de Souza Departamento de Informática - PUC-Rio

Prof. Hugo Fuks

Departamento de Informática - PUC-Rio

Prof $^{\mathrm{a}}$. Ana Cristina Bicharra Garcia Departamento de Ciência da Computação - UFF

Prof ${ }^{\mathrm{a}}$. Simone Bacellar Leal Ferreira Departamento de Informática Aplicada - UNIRIO

Prof. José Eugenio Leal

Coordenador Setorial do Centro Técnico Científico - PUC -Rio

Rio de Janeiro, 27 de agosto de 2010. 
Todos os direitos reservados. É proibida a reprodução total ou parcial do trabalho sem autorização da universidade, do autor e dos orientadores.

\section{José Eurico de Vasconcelos Filho}

Graduou-se em Informática na Universidade de Fortaleza (UNIFOR), no ano de 2001. Obteve o título de Mestre em Informática no Mestrado em Informática Aplicada da UNIFOR, em 1994. É professor assistente da Faculdade Integrada do Ceará - FIC onde leciona disciplinas na área de Inteligência Artificial e Interação Humano-Computador. É pesquisador na área de Simulação Multi-Agente, Mapeamento Colaborativo e Interação Humano-Computador no Laboratório de Engenharia do Conhecimento da UNIFOR.

Ficha Catalográfica

Vasconcelos Filho, José Eurico de

Um modelo de suporte ao design baseado no rationale: relacionando espaço de problema ao espaço de solução no design / José Eurico de Vasconcelos Filho ; orientadora: Simone Diniz Junqueira Barbosa. - 2010.

124 f.: il. (color.) ; $30 \mathrm{~cm}$

Tese (doutorado)-Pontifícia Universidade Católica do Rio de Janeiro, Departamento de Informática, 2010.

Inclui bibliografia

1. Informática - Teses. 2. Design baseado em modelos. 3. Design rationale. 4. Rastreabilidade de requisitos. 5. Interação humano-computador. I. Barbosa, Simone Diniz Junqueira. II. Pontifícia Universidade Católica do Rio de Janeiro. Departamento de Informática. III. Título. 
A meu Senhor e a meus pais. 


\section{Agradecimentos}

À minha família, por existirem e estarem sempre perto.

À minha esposa, por seu amor e apoio incondicional durante todo esse processo.

A meu amigo e orientador Vasco Furtado que sempre me indicou um norte e acreditou em meu potencial.

À minha orientadora Simone Barbosa, por me tranquilizar ao leme da nau.

A todos os colegas, amigos e professores que direta ou indiretamente me auxiliaram durante esta empreitada. 


\section{Resumo}

Vasconcelos Filho, José Eurico de; Barbosa, Simone Diniz Junqueira; Furtado, João José Vasco. Um Modelo de Suporte ao Design Baseado no Rationale: Relacionando Espaço de Problema ao Espaço de Solução no Design, Modelagem, Design Rationale. Rio de Janeiro, 2010. 124 p. Tese de Doutorado - Departamento de Informática, Pontifícia Universidade Católica do Rio de Janeiro.

O design de sistemas interativos é um processo complexo, iterativo e colaborativo, que requer diferentes conhecimentos para sua realização, e composto por diferentes atividades que se inter-relacionam na composição de um produto final. As informações produzidas durante cada atividade são de extrema relevância para a continuidade do processo e para a compreensão, explicação e manutenção do produto produzido. Estas informações devem estar relacionadas e integradas em um discurso claro e coerente, permitindo sua rastreabilidade e contemplando a proveniência e o contexto em que foram produzidas. Observa-se, entretanto, que as propostas de captura e modelagem do processo de design em IHC provêem uma visão fragmentada e/ou incompleta deste processo. Por esta razão, propõe-se aqui um modelo epistêmico, baseado em Design Rationale (DR), adequado ao registro e modelagem do design de sistemas interativos. Com base na revisão e análise das principais visões e propostas do processo de design, seus requisitos, modelos, notações, linguagens de suporte foi proposto o modelo epistêmico Ideia. O modelo tem como principal objetivo registrar e comunicar as atividades de design de forma coesa, oferecendo subsídios para a reflexão da equipe de design sobre as atividades do processo de design, bem como informações de proveniência para a rastreabilidade das informações. Para isso, o modelo propõe a integração dos insumos e produtos (e.g., requisitos, modelos, artefatos) das atividades de design, oferecendo uma ponte entre as atividades de análise (espaço de problema) e de design conceitual (espaço de solução) por meio do DR. O modelo foi implementado no protótipo Deprost e adotado no design de parte de um sistema colaborativo, o sistema WikiMapps, possibilitando avaliar a proposta através de um estudo de caso sobre seu desempenho quanto ao que é proposto.

\section{Palavras-chave}

Design Baseado em Modelos; Design Rationale; Rastreabilidade de Requisitos; Interação Humano-Computador. 


\section{Abstract}

Vasconcelos Filho, José Eurico de; Barbosa, Simone Barbosa Junqueira (advisor); Furtado, João José Vasco (co-advisor). A Design Model Based on Rationale: Relating Problem Space to Solution Space on Design, Interactive System Design, Design Rationale. Rio de Janeiro, 2010. 124 p. DSc. Thesis - Departamento de Informática, Pontifícia Universidade Católica do Rio de Janeiro.

The design of interactive systems is a complex, iterative and collaborative process, composed of different activities that are interrelated in the composition of a final product and requires different expertise to perform it. The information produced during each activity is very important for the continuity of the process and to understand, explain and maintain the product produced. This information must be related and integrated in a clear and coherent speech, allowing traceability and addressing the origin and the context in which they were produced. It is noted, however, that the proposed process catch and modeling in HCI design provide a fragmented and/or incomplete view of this process. For this reason, we propose an epistemic model, based on Design Rationale (DR), suitable for the recording and modeling of the design of interactive systems. Based on the review and analysis of the key views and proposals of the design process, their requirements, models, notations and support languages, the epistemic model Ideia has been proposed. The model has as its main goal to register and report design activities in a cohesive manner, offering support for the reflection of the design team about the activities of the design process as well as information sources for the traceability of information. For this, the model proposes the integration of inputs and outputs (e.g., requirements, models, artifacts) of the activities of analysis (problem space) and the conceptual design (solution space) DR. The model was implemented in the Deprost prototype and adopted in the design of part of the WikiMapps project, allowing us to evaluate the proposal in a real case study.

\section{Keywords}

Model-Based Design; Design Rationale; Requirements Traceability; Human-Computer Interaction. 
1 INTRODUÇÃO

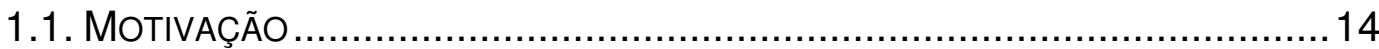

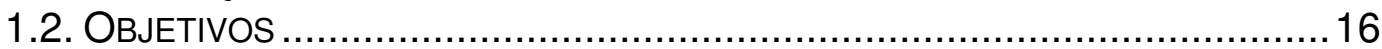

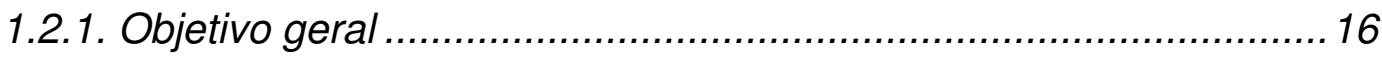

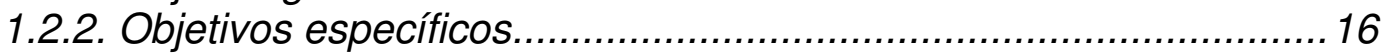

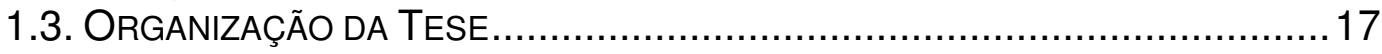

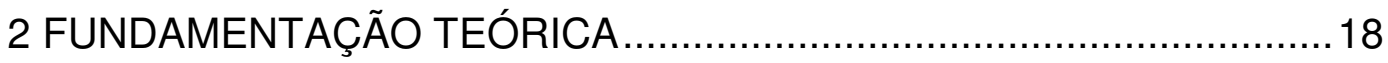

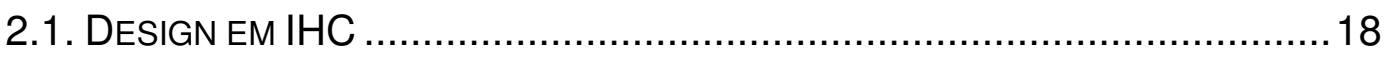

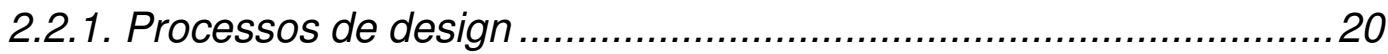

2.2. REPRESENTAÇÕES, MODELOS E ABORDAGENS DE DESIGN EM IHC ..........25

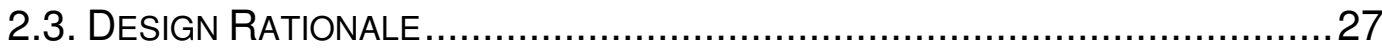

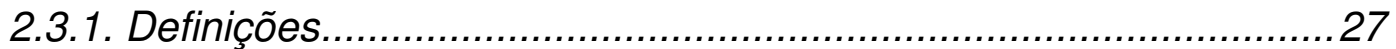

2.3.2. Motivações para o Design Rationale ...........................................28

2.3.3. Critérios de caracterização de abordagens de DR........................29

2.3.4. Design Rationale e os Espaços de Design.....................................31

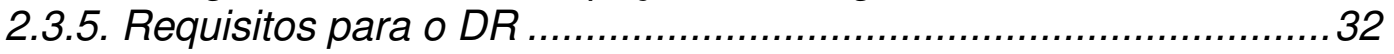

2.3.6. Design Rationale como Ferramenta Epistêmica ............................ 33

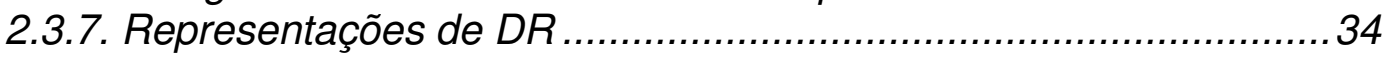

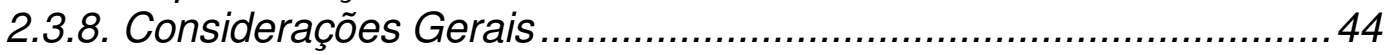

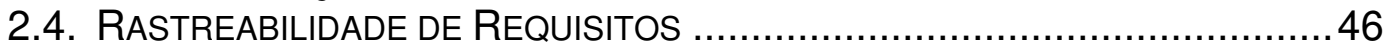

2.5. PROVENIÊNCIA DE DADOS (PROVENANCE) .......................................... 48

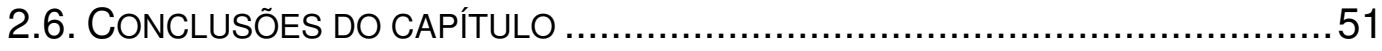

3 IDEIA: UM MODELO BASEADO NO RATIONALE COMO SUPORTE AO PROCESSO DE DESIGN ........................................ 53

3.1. QUESTÕES ENDEREÇADAS PELO MODELO ………............................. 53

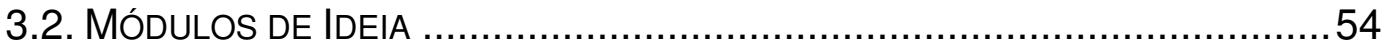

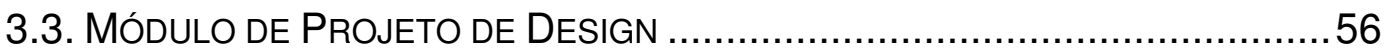

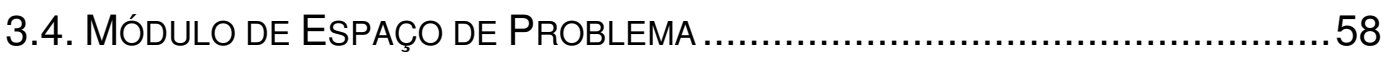

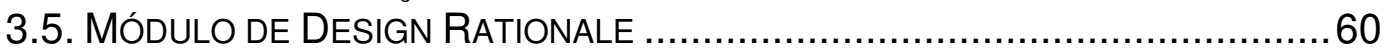

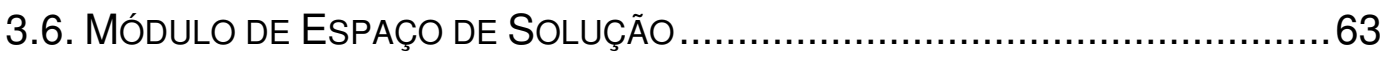

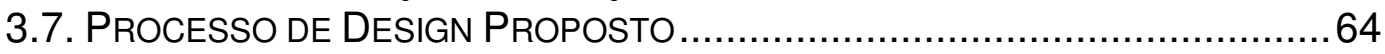

3.8. EXEMPLO DE SUPORTE AO REGISTRO DO DESIGN COM IDEIA...................6 65

3.8.1. Passo 1) O início do projeto ...................................................6

3.8.2. Passo 2) Identificando necessidades e estabelecendo

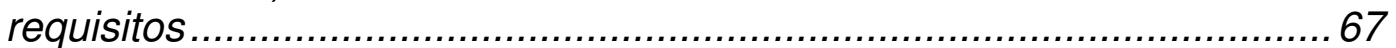

3.8.3. Passo 3) Desenvolvendo design alternativos que auxiliem os usuários a cumprir seus objetivos no sistema. .................................. 71

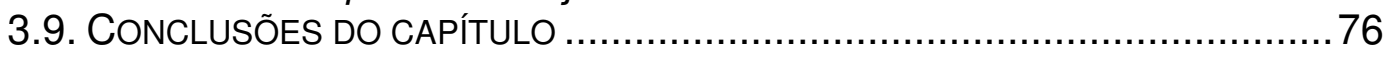

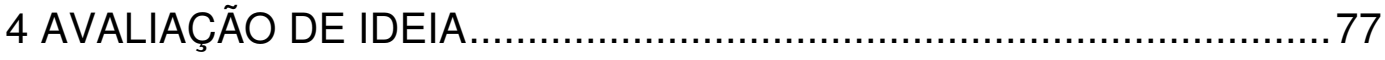

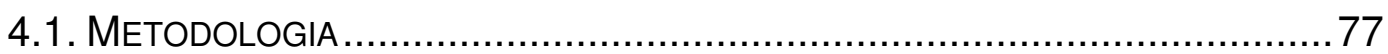

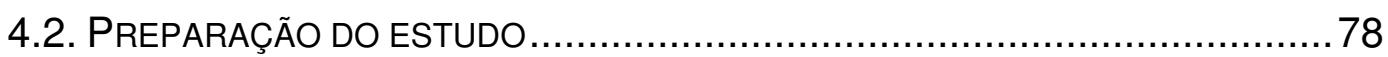

4.2.1. Definição das questões de investigação do estudo........................ 78 
4.2.2. Definição dos critérios de avaliação das questões de

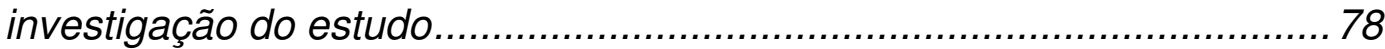

4.2.3. Desenvolvimento do Protótipo Deprost ....................................... 79

4.2.4. Definição do Caso .............................................................. 83

4.2.5. Participantes, local de realização do estudo e instrumentos de coleta de dados ............................................................................ 84

4.2.6. Processo e cronograma de execução do estudo de caso............... 85

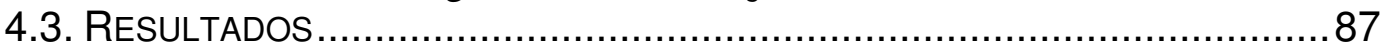

4.3.1. Observações gerais registradas durante o processo de

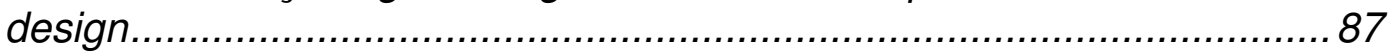

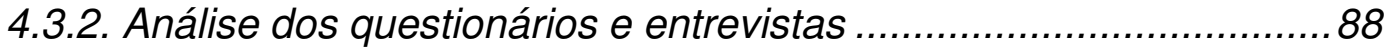

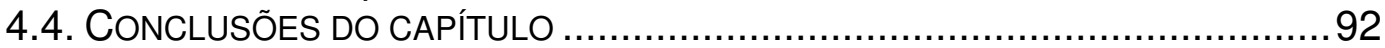

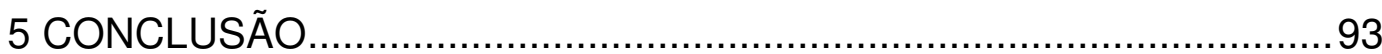

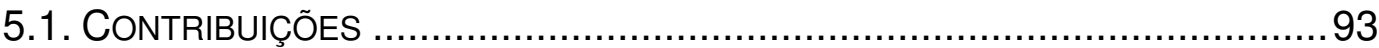

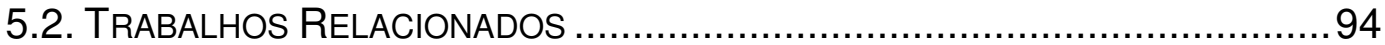

5.2.1 Suporte à rastreabilidade .........................................................94

5.2.2 Suporte à documentação e rationale do processo de design...........95

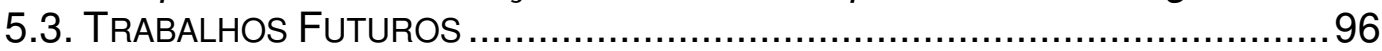

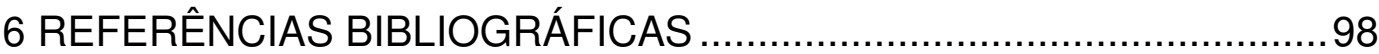

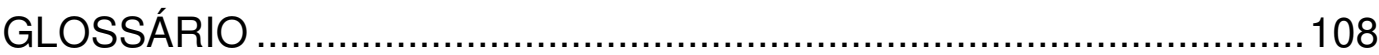

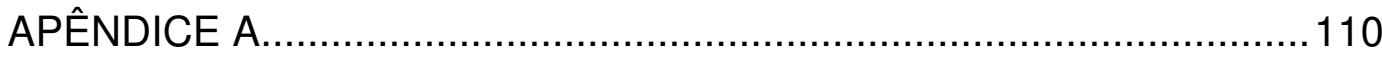

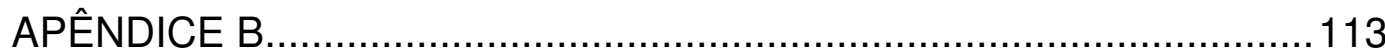




\section{Lista de Figuras}

FIGURA 2.1: DESIGN É UM PROCESSO INTERPRETATIVO ONDE NÃO HÁ VERDADES ÚNICAS OU ABSOLUTAS. (FIGURA ADAPTADA DE PREECE

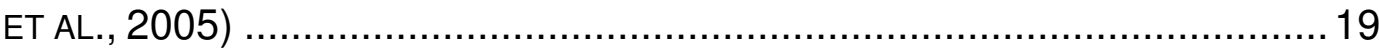

FIGURA 2.2: O PROCESSO DE DESIGN DE INTERAÇÃO POR NÄHR (2009). ........20

FIGURA 2.3: PROCESSO DE DESIGN DE INTERAÇÃO PROPOSTO POR

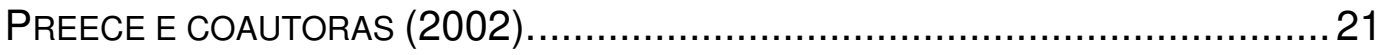

FiguRA 2.4: MOdELO ESTRELA DE HIX E HARTSON (1989)....................... 22

FIGURA 2.5: PROCESSO DE DESIGN DE INTERAÇÃO PROPOSTO POR DIX E COAUTORES. TRADUZIDA DE DIX ET AL (2004). .................................. 23

Figura 2.6: DESign RATIONALE NO PROCESSO DE DESIGN, LIGANDO REQUISITOS E DESIGN. TRADUZIDA DE (BURGE E BROWN, 2000)

FIGURA 2.7: EXEMPLO DE ARGUMENTAÇÃO BASEADO NA ESTRUTURA PROPOSTA POR TOULMIN. ADAPTADA DE TOULMIN (1958).

FIGURA 2.8: ElEMENTOS E RELAÇÕES DA NOTAÇÃO IBIS. AdAPTADA DE KUNZ E RITTEL (1970).

FIGURA 2.9: ElEMENTOS E RELAÇÕES DO MODELO PHI. AdAPTADA DE MCCALL (1991)

Figura 2.10: EleMENTOS dO MODELO POTTS \& BRUNS. AdAPTADA DE POTTS \& BRUNS (1988)

FIGURA 2.11: VoCABULÁRIO DE DRL, FONTE: MEDEIROS (2006). 38

FIGURA 2.12: O VOCABULÁRIO DE QOC. AdAPTADA DE MACLEAN E COAUTORES (1991).

FIGURA 2.13: ELEMENTOS DO VOCABULÁRIO DE KUABA. FONTE:

MEDEIROS (2006).

FIGURA 2.14: CICLO DE DESIGN PROPOSTO POR LOURIDAS E LOUCOPOLOS (2000). 44

FIGURA 2.15: ONTOLOGIA DE PROVENIÊNCIA W7 (RAM \& LIU, 2007)............. 50

FIGURA 3.1: MódULOS DO MODELO. ............................................... 55

FIGURA 3.2: ELEMENTOS DE RELACIONAMENTO ENTRE MÓDULOS. 56 
Figura 3.3: Elementos do Módulo de Contexto de Design e suAS RELAÇÕES.

FIGURA 3.4: ELEMENTOS E RESPECTIVOS RELACIONAMENTOS DO MÓDULO DE ESPAÇO DE PROBLEMA 60

FiguRA 3.5: ElEMENTOS E RELAÇÕES dO MÓdULO dE DESIGN

RATIONALE. 62

FIGURA 3.6: ElEMENTOS E RELAÇÕES (INTERNAS) DO MODELO DE ESPAÇO DE SOLUÇÃO. 64

Figura 3.7: Processo de Design Integrado. Adaptada de Preece e COAUTORAS (2005) E LOURIDAS E LOUCOPOLOS (2000). 65

FIGURA 3.8: EXEMPLO DE INSTÂNCIA DO MÓDULO DE PROJETO DE DESIGN. .....67

FIGURA 3.9: EXEMPLO DE INSTÂNCIA DO MÓDULO DE ESPAÇO DE PROBLEMA NO EXEMPLO YOURMUSICSTORE. 70

FIGURA 3.10: REGISTRO DE UMA QUESTÃO DE DESIGN ORIGINADA DA ANÁLISE DE UM DOS ELEMENTOS DE ANÁLISE REGISTRADOS.. 71

FIGURA 3.11: PARTE DO PROCESSO DE DISCUSSÃO SOBRE A QUESTÃO REGISTRADA. 72

FIGURA 3.12: TOMADA DE DECISÃO SOBRE UMA PROPOSTA. 73

FIGURA 3.13: ASSOCIAÇÃO ENTRE A PROPOSTA DE DESIGN E UM ELEMENTO QUE DELA RESULTA 73

FIGURA 3.14: QUESTÃO DE DESIGN CONTRASTANDO UMA PROPOSTA DE ELEMENTO DE DESIGN E UM ELEMENTO DE ANÁLISE COM UM OBJETIVO DO USUÁRIO.

FIGURA 3.15: ALTERAÇÃO DE UM ELEMENTO DE DESIGN A PARTIR DA REFLEXÃO SOBRE OUTROS ELEMENTOS REGISTRADOS NO MODELO. 74

FIGURA 3.16: EXEMPLO DE INSTÂNCIA DO MODELO NO EXEMPLO

YOURMUSICSTORE. 75

FIGURA 4.1: TELA PRINCIPAL (PERFIL ADMINISTRADOR) DO PROTÓTIPO DEPROST. 80

FiguRA 4.2: TELA do Projeto de DESIGN. ......................................... 81

FiguRA 4.3: TELA DE DESIGN RATIONALE............................................. 83

FIGURA 4.4: EXEMPLO DE UM MAPA COLABORATIVO DESENVOLVIDO EM WIKIMAPPS. 


\section{Lista de Abreviaturas}

ACD - Activity-Centered Design

ADD - AUGMENTING DESIGN DOCUMENTATION

CLG - COMMAND-LANGUAGE GRAMMAR

CTT - CONCUR TASK TREES

DQN - DESIGN QUESTION NOTATION

DR - DESIGN RATIONALE

DRIM - Design ReCOMmendation AND INTENT MOdEL

DRL - DECISION REPRESENTATION LANGUAGE

ENGSEM - ENGENHARIA SEMIÓTICA

GIBIS - GRAPHICAL ISSUE-BASED INFORMATION SYSTEM

GTA - GROUPWARE TASK ANALYSIS

GR - GEREANCIAMENTO DE REQUISITOS

HCD - HUMAN CENTERED DESIGN

HTA - HIERARCHICAL TASK ANALYSIS

IBIS - ISSUE-BASED INFORMATION SYSTEM

IHC - INTERAÇÃO HUMANO-COMPUTADOR

IMML - INTERACTIVE MESSAGE MODELING LANGUAGE

INFORAT - INFERENCING OVER RATIONALE

ITIBIS - INDENTED TEXT ISSUE-BASED INFORMATION SYSTEM

MAD - MÉTHOde ANALYTIQUE dE DESCRIPTION

MOLIC - MODELING LANGUAGE FOR INTERACTION AS CONVERSATION

PHI - Procedural HiERARCHY OF ISSUES

QOC - QUESTIONS, OPTIONS, AND CRITERIA

REMAP - REPREsentATION AND MAINTENANCE Of PROCESS KNOWLEDGE

RR - RASTREABILIDADE DE REQUISITOS

SBD - SCENARIO BASED DESIGN

TAG - TASK-ACTION GRAMMAR

TKS - TASK KNOWLEDGE STRUCTURES

UAN - USER ACTION NOTATION

UCD - USER CENTERED DESIGN 